\title{
RENDIMIENTO Y CALIDAD DEL FRUTO DE DURAZNERO BI-VARIETAL EN UN SISTEMA MIAF EXPUESTO A HELADAS
}

\author{
YIELD AND FRUIT QUALITY OF BI-VARIETAL PEACH TREES \\ IN A MIAF SYSTEM EXPOSED TO FROSTS
}

\author{
Horacio Santiago-Mejía', José I. Cortés-Flores ${ }^{2}$, Antonio Turrent-Fernández ${ }^{4}$, \\ Rocio Albino-Garduño ${ }^{1}$, Victor H. Volke-Haller ${ }^{2}$ e Hilda A. Zavaleta-Mancera ${ }^{3}$
}

\begin{abstract}
'Universidad Intercultural del Estado de México, San Felipe del Progreso, Estado de México, México. ${ }^{2}$ Colegio de Postgraduados (CP), Campus Montecillo, Edafología, Montecillo, Estado de México, México. ${ }^{3} \mathrm{CP}$, Campus Montecillo, Botánica, Montecillo, Estado de México, México. ${ }^{4}$ Instituto Nacional de linvestigaciones Forestales, Agrícolas y Pecuarias, Campo Experimental Valle de México, Coatlinchán, Texcoco, Estado de México, México.
\end{abstract}

*Autor de correspondencia (rocio.albino@uiem.edu.mx)

\section{RESUMEN}

El duraznero es un componente estratégico de la Milpa Intercalada con Árboles Frutales (MIAF) por el alto precio del fruto en épocas de escasez, en la que su producción es limitada por el daño que le causan las heladas. El patrón de floración del duraznero es acrópeto y las temperaturas en una helada de radiación son más bajas en el estrato inferior del árbol, lo que lleva a usar árboles compuestos por una variedad de floración tardía (Vft) en el estrato inferior y una de floración precoz (Vfp) en el superior (Cbi-v). El objetivo fue evaluar el rendimiento y calidad de fruto del duraznero en respuesta a la composición varietal del árbol y a la fertilización de dos Vfp y dos Vft en la MIAF en un ambiente con incidencia de heladas. El experimento se estableció en el Campo Experimental Valle de México del INIFAP en el año 2005 y se evaluó en 2012. Con un diseño factorial $2^{5}$ lotificado a $1 / 4$ se analizó la respuesta en dos niveles de los factores composición varietal, fertilizante mineral y pollinaza. Las variables evaluadas fueron parámetros de rendimiento y calidad del fruto, en las que se hizo análisis de varianza, comparación de medias y regresión. La combinación de la Vfp Puebla injertada en la Vft Supremo tuvo el mayor rendimiento $\left(15.1 \mathrm{~kg} /\right.$ árbol) y eficiencia del rendimiento $\left(1.46 \mathrm{~kg} \mathrm{~cm}^{-2}\right)$. La fertilización (90-30-90 g N-P $\mathrm{O}_{5}-\mathrm{K}_{2} \mathrm{O} /$ árbol/año) incrementó los sólidos solubles totales (SST) en los frutos de Supremo (de 11.1 a $12.6^{\circ} \mathrm{Brix}$ ). La pollinaza (3 kg/árbol/año) incrementó los SST en los frutos de Supremo y disminuyó la firmeza y acidez en los de Puebla en la composición bi-varietal. El manejo de la Cbi-v y fertilización mejoró el rendimiento y la calidad de fruto de durazno en la MIAF en ambientes con incidencia de heladas de radiación.

Palabras clave: Prunus persica (L.) Batsch, MIAF, NPK, pollinaza, variedad precoz, variedad tardía.

\section{SUMMARY}

The peach tree is an strategic component of the Milpa Intercropped with Fruit Trees (MIAF) due to the high price of the fruit in times of scarcity, when its production is limited by damage due to frosts. The peach tree bloom is acropetal and the temperature in a radiation frost is lower in the lower stratum of the tree, what led us to use trees composed of a cultivar of late-flowering (Vft) in the lower stratum and one of early-flowering ( $\mathrm{Vfp})$ in the upper one (Cbi-v). The aim was to evaluate yield and fruit quality of the peach in response to the varietal composition of the tree and to fertilization of two Vfp and two $\mathrm{Vft}$ in the MIAF in an environment with frosts incidence. The experiment was established at the Valle de México Experiment Station of INIFAP in 2005 and it was evaluated in 2012. The response at two levels of the factors varietal composition, mineral fertilizer and chicken manure was evaluated through a
$2^{5}$ factorial design lotified to $1 / 4$. The traits measured were parameters of yield and fruit quality, on which analysis of variance, comparison of means and regression were performed. The combination of the Vfp Puebla grafted on the Vft Supremo had the highest yield $(15.1 \mathrm{~kg} /$ tree $)$ and yield efficiency $(1.46 \mathrm{~kg}$ $\mathrm{cm}^{-2}$ ). The fertilization (90-30-90 g N-P205-K20/tree/year) increased total soluble solids (SST) in Supremo fruits (from 11.1 to $12.6^{\circ} \mathrm{Brix}$ ). The chicken manure ( $3 \mathrm{~kg} /$ tree/year) increased the SST in fruits of Supremo and decreased the firmness and acidity in those of Puebla in the bi-varietal composition. The management of the $\mathrm{Cbi}-\mathrm{v}$ and fertilization improved the quality and peach fruit yield in the MIAF in environments with radiation frost incidence.

Index words: Prunus persica (L.) Batsch, chicken manure, earlyflowering cultivar, late-flowering cultivar, MIAF, NPK.

\section{INTRODUCCIÓN}

En el altiplano mexicano, el árbol de durazno [Prunus persica (L.) Batsch], de variedad precoz e intermedia, es un componente estratégico para la Milpa Intercalada con Árboles Frutales (MIAF) por el alto precio del fruto en épocas de escasez; sin embargo, en esta región, la cosecha es incierta por daños causados por heladas durante el invierno y el inicio de la primavera cuando ocurre la floración y el amarre de fruto. El patrón de floración del duraznero es de la base hacia el ápice (acrópeto) (SantiagoMejía et al., 2015); ésto implica que durante las heladas de radiación, las flores con mayor riesgo de daño son las de la parte inferior del dosel, pues la temperatura del aire es más fría que en la parte superior (Lhomme et al., 2007). Las investigaciones en injertos e interinjertos de duraznero se han enfocado en mejorar la calidad de los frutos (Webster y Palmer, 2017), incluyendo la firmeza (Iglesias et al., 2019), el color (Marcon et al., 2019), sólidos solubles totales y rendimiento (Font i Forcada et al., 2019). La fertilización mineral (Minas et al., 2018; Rubio et al., 2020) y estiércol (Narayan et al., 2016) en el duraznero incrementan el rendimiento y calidad del fruto; sin embargo, el efecto de la composición bi-varietal del árbol, usando variedades precoces y tardías y la fertilización no han sido evaluados 
conjuntamente, ni comparados con la composición univarietal. El objetivo de esta investigación fue analizar el rendimiento, la eficiencia del rendimiento y la calidad de fruto de durazneros de floración tardía y precoz en su composición bi-varietal y uni-varietal, fertilizados con NPK y pollinaza en el sistema MIAF en un ambiente con incidencia de heladas de radiación.

\section{MATERIALES Y MÉTODOS}

\section{Sitio y diseño experimental}

El experimento se estableció con el sistema MIAF en el Campo Experimental Valle de México del INIFAP (19०29' $17^{\prime \prime} \mathrm{N}$ y $\left.98^{\circ} 53^{\prime} 41^{\prime \prime} \mathrm{O}, 2280 \mathrm{msnm}\right)$, Texcoco, Estado de México, en el año 2005. El clima es templado seco, con temperatura media anual de $15.45^{\circ} \mathrm{C}$, precipitación media anual de $644 \mathrm{~mm}$ y el suelo es Fluvisol móllico (Govaerts et al., 2008).

El diseño de tratamientos fue un factorial $2^{5}$ (32 tratamientos) en un arreglo de parcelas divididas, lotificado a $1 / 4$. Los cinco factores y sus dos niveles fueron: 1) composición varietal del árbol (Cva), bi-varietal y univarietal; 2) variedad de floración tardía (Vft) Supremo y Zacatecas; 3) variedad de floración precoz (Vfp) CP-Precoz y Puebla; 4) fertilización (F) N- $\mathrm{P}_{2} \mathrm{O}_{5}-\mathrm{K}_{2} \mathrm{O}, 60-15-60$ y 9030-90 g/árbol/año; 5) pollinaza (P), 0 y 3 kg/árbol/año. El método de lotificación se realizó de acuerdo con la teoría de los campos de Galois (Kempthome, 1979). Los contrastes de definición para lotificar a $1 / 2$ fueron el factor Cva y el otro contraste para lotificar a $1 / 4$ fue la interacción de cuatro factores $(V f t \times V f p \times F \times P)$. La lista de 32 tratamientos, con una repetición, quedó fraccionada en cuatro lotes (como parcela grande) de ocho tratamientos cada uno (parcela chica) (Santiago-Mejía et al., 2015).

\section{Establecimiento y manejo del experimento}

Los porta-injertos se trasplantaron en mayo de 2005 en hileras separadas a $14.4 \mathrm{~m}$, con orientación norte-sur y a $1.0 \mathrm{~m}$ de distanica entre plantas. Los durazneros univarietales se plantaron de manera alterna; es decir, un árbol completo de la variedad de floración tardía (Supremo o Zacatecas) con uno de la variedad de floración precoz (CP-Precoz o Puebla). Los durazneros bi-varietales fueron árboles compuestos por dos variedades, floración tardía y precoz; la tardía se injertó en el estrato inferior y la precoz sobre ésta en el estrato superior. Para los durazneros univarietales y bi-varietales, el injerto sobre el porta-injerto criollo se realizó a los $15 \mathrm{~cm}$ del nivel del suelo en abril de 2006 y en los durazneros bi-varietales el injerto de la variedad precoz sobre la tardía se hizo a $1.5 \mathrm{~m}$ de altura del árbol en marzo de 2007. La conducción de los árboles fue a través del sistema "Tatura" modificado (SantiagoMejía et al., 2015). La unidad experimental fue de cuatro árboles y la parcela útil de los dos árboles centrales: dos árboles bi-varietales o dos árboles uni-varietales. Tanto el fertilizante como la pollinaza se aplicaron en abril y agosto de cada año sobre la hilera de árboles a $1 \mathrm{~m}$ de distancia del tronco, en cepas de $20 \mathrm{~cm}$ de profundidad. El experimento se mantuvo sin restricción de humedad con riegos por gravedad.

La incidencia de heladas fue registrada del 17 de noviembre de 2011 al 31 de marzo de 2012; para ello, se midió la temperatura del aire a $1.5 \mathrm{~m}$ de altura del dosel del árbol con una microestación WatchDog 1000 Series (Spectrum Technologies, Inc.; Fort Worth, TX, EUA) cada 15 minutos. La floración y el amarre de fruto, que dio origen a la cosecha del ciclo 2012, estuvieron expuestas a cinco heladas de radiación intensas $\left(-2.1\right.$ y $\left.-8.1{ }^{\circ} \mathrm{C}\right)$ del 29 de noviembre al 03 de diciembre de 2011, cuatro de intensidad intermedia $\left(-1.1\right.$ a $\left.-2.0^{\circ} \mathrm{C}\right)$ del 11 al 19 de diciembre de 2011 y seis de baja intensidad $\left(0.0\right.$ a $\left.-1.0^{\circ} \mathrm{C}\right)$ del 12 de diciembre de 2011 al 12 de enero de 2012. En 22 ocasiones la temperatura estuvo entre 1 y $5{ }^{\circ} \mathrm{C}$, del 25 de noviembre de 2011 al 21 de marzo de 2012.

\section{Variables evaluadas}

En el año 2012 se evaluó el rendimiento de fruto, la eficiencia del rendimiento (ER) y la calidad del fruto, usando los dos árboles de la parcela útil en cada tratamiento. La cosecha de los frutos se realizó en madurez fisiológica (60 $\%$ de chapeado en la superficie del pericarpio). El rendimiento se determinó con el número de frutos por árbol y la suma del peso de cada fruto ( $\mathrm{kg} / \mathrm{a} r \mathrm{bol})$; para ello se usó una balanza (Mettler Toledo PC 2000, Columbus, Ohio, EUA). La ER se calculó con la fórmula proporcionada por Westwood (1993), que considera la relación entre el rendimiento y el área de la sección transversal del tronco del árbol $\left(\mathrm{kg} \mathrm{cm}^{-2}\right.$ del área del tronco). El diámetro del tronco se midió $5 \mathrm{~cm}$ arriba de la unión del injerto con el porta-injerto con un pie de rey digital de precisión de $0.01 \mathrm{~cm}$ (Truper ${ }^{\circledR}$ COD 14388, México). Para la calidad del fruto se tomaron al azar seis frutos de cada árbol de la parcela útil; a ellos se les midió el tamaño (diámetro polar y ecuatorial en $\mathrm{cm}$ ), con un pie de rey digital de precisión de $0.01 \mathrm{~cm}$ (Truper ${ }^{\circledR}$ COD 14388, México); firmeza $\left(\mathrm{kg} \mathrm{cm}^{-2}\right)$ con un texturómetro (Chatillon, modelo FDV-30, Wagner Instruments, Greenwich, CT, EUA) con un puntal de $8 \mathrm{~mm}$ de diámetro y $8 \mathrm{~mm}$ de profundidad de acuerdo con el método de Kader y Mitchel (1989); sólidos solubles totales (SST en ${ }^{\circ}$ Brix) con un refractómetro digital (PR-100 ATAGO, Tokio, Japón); acidez titulable (\% ácido málico) con el método propuesto por la AOAC (1990) y color del pericarpo ('Hue) con un colorímetro por reflexión (HunterLab D25, Reston, Virginia, EUA) de acuerdo con la metodología de Little (1975). 


\section{Análisis estadístico}

Para conocer el efecto de los factores de estudio, los datos se analizaron de dos maneras: 1) considerando la respuesta acumulada de las variedades con floración precoz más la tardía, lo cual explica el fenómeno general, pero oculta el efecto de cada variedad y 2) a las Vfp y Vft por separado, lo que permite explicar el aporte de las variedades con cada tipo de floración. Para determinar la respuesta de las variables dependientes a los cinco factores (Cva, Vft, Vfp, F y P) se realizó el análisis de varianza correspondiente al diseño experimental ( $P \leq$ 0.05 y $\mathrm{P} \leq 0.01)$ y análisis de regresión mediante el procedimiento de selección a pasos o stepwise (sle = 0.10 , sls $=0.05$ y variables mudas o dummy, que permiten conocer el efecto de cada factor de estudio) usando SAS ${ }^{\oplus}$ versión 9. En el análisis de varianza las interacciones de tres y cuatro factores se consideraron como parte del error experimental. Los efectos significativos de los factores de estudio se graficaron para apoyar la interpretación de las respuestas observadas; los gráficos se elaboraron con SigmaPlot versión 11.0. Así, la respuesta de las variedades se analizó con tres ecuaciones diferentes: tardía, precoz y una tercera ecuación con la suma de ambas.

\section{RESULTADOS Y DISCUSIÓN}

\section{Rendimiento y eficiencia del rendimiento}

La Vfp Puebla rindió más que CP-Precoz. El mayor rendimiento de Puebla (9.62 kg/árbol) ocurrió cuando se injertó sobre Supremo (composición bi-varietal) (Figura 1A). La eficiencia de rendimiento en la Vfp Puebla fue mayor en la composición bi-varietal que en la uni-varietal
(Figura 2A) y se incrementó aún más cuando se combinó con la Vft Supremo (Figura 1B). Así, la combinación Puebla y Supremo alcanzaron el mayor valor de ER $\left(1.46 \mathrm{~kg} \mathrm{~cm}^{-2}\right)$; ésto significa que con diámetros menores del tronco en la composición bi-varietal el rendimiento fue mayor con respecto a los troncos más gruesos en la composición univarietal. Esta ER generó un rendimiento de $11.45 \mathrm{~kg} / \mathrm{a} r b o l$ con la composición bi-varietal Supremo Puebla, aportando 6.70 y $4.75 \mathrm{~kg}$, respectivamente; además, Supremo aumentó la eficiencia de rendimiento con la fertilización alta (90-30$90 \mathrm{~g}$ de $\mathrm{N}-\mathrm{P}_{2} \mathrm{O}_{5}-\mathrm{K}_{2} \mathrm{O} / a$ rbol) (Figura 2B). En la composición bi-varietal, las $\mathrm{Vft}$ funcionaron como interinjertos; en este caso, a Supremo se le atribuye parte del incremento en la eficiencia del rendimiento. Al respecto, se ha reportado que el uso de interinjertos regula la competencia entre el crecimiento vegetativo y el reproductivo (Marra et al., 2013), incrementa la producción de fruto por unidad de área transversal del tronco y favorece el abastecimiento de fotosintatos de reserva a la raíz (Mestre et al., 2017; Yano et al., 2002); además, el rendimiento y la ER de duraznero se incrementan con dosis adecuadas de fertilización NPK (Minas et al., 2018; Rubio Ames et al., 2020).

Zacatecas indujo a menos frutos en la composición bivarietal, de las dos $\vee f p$, en comparación de la uni-varietal (Figura 3A). La mejor combinación agronómica del árbol fue la Vft Supremo combinada con la Vfp Puebla, en la composición bi-varietal; en ésta se alcanzó el mayor rendimiento total con 5.49 y $9.62 \mathrm{~kg} /$ árbol, para Supremo y Puebla, respectivamente. En esta composición bi-varietal Puebla tuvo mayor número de frutos (Figura $3 \mathrm{~A}$ ) y peso de frutos (Figura 3B), en tanto que en la composición univarietal Puebla tuvo el mayor número de frutos (cuando se alternó con Zacatecas) (Figura 3A), pero el peso (Figura
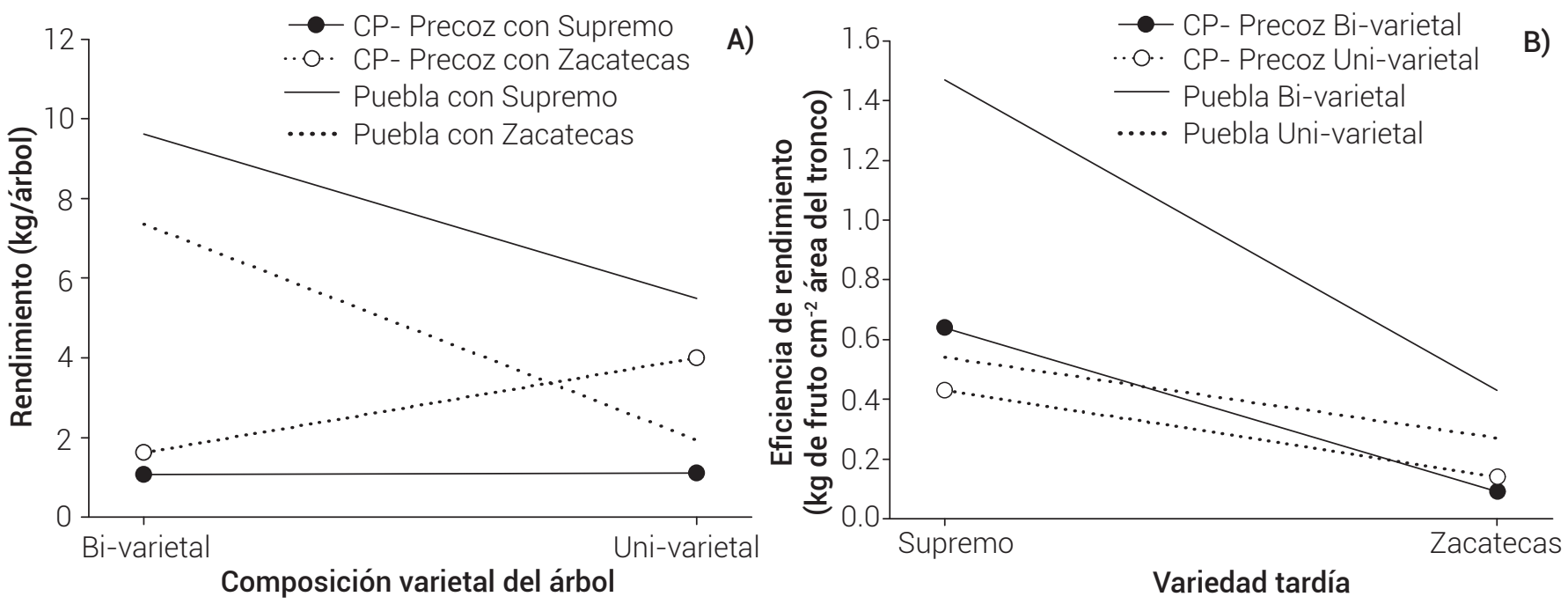

Figura 1. Efecto de la interacción de las variedades con floración precoz, CP Precoz y Puebla: A) con la composición varietal del árbol en el rendimiento de fruto y B) con las variedades de floración tardía en la eficiencia total del rendimiento. 


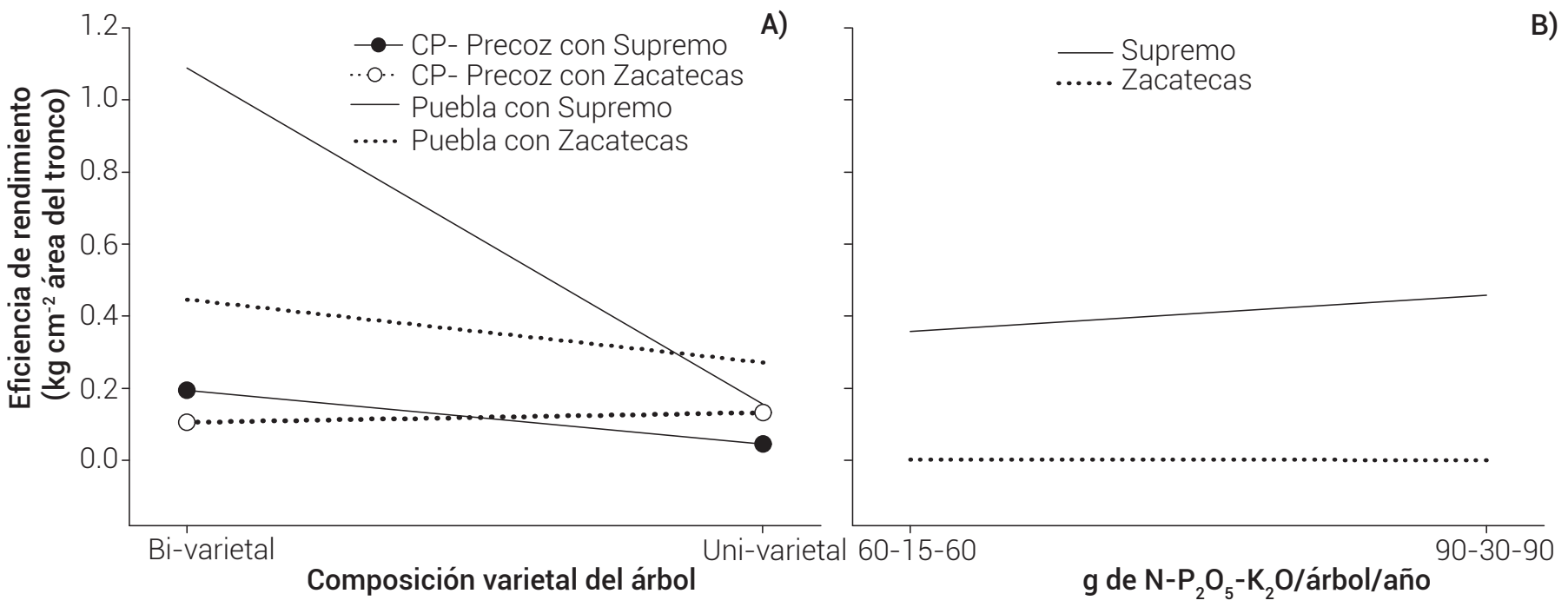

Figura 2. Efecto en la eficiencia de rendimiento de duraznero, A) de la interacción composición varietal del árbol con la variedad tardía en las variedades precoces y $\mathrm{B}$ ) de la fertilización $\mathrm{N}-\mathrm{P}_{2} \mathrm{O}_{5}-\mathrm{K}_{2} \mathrm{O}$ en las variedades tardías.

3B) y el diámetro (Cuadro 1) del fruto fueron menores.

\section{Calidad del fruto}

Los frutos con mayor firmeza se obtuvieron con Puebla en composición bi-varietal o uni-varietal alternada con Zacatecas (Figura 4A). Debido a que Zacatecas no tuvo producción de fruto, la fertilización de NPK y estiércol pudo aprovecharse únicamente por los frutos de Puebla. La literatura confirma que el contenido de nutrimentos en las plantas está influenciado por el inter-injerto, pues mejora la distribución de fotoasimilados, nutrientes y hormonas, ya que disminuyen la competencia entre el crecimiento vegetativo y el reproductivo (Marcon Filho et al., 2019). En este estudio, la firmeza de los frutos de las Vft sólo pudo incrementarse usando a Supremo ya que no hubo efecto de los otros factores estudiados (Cuadro 1). La firmeza de los frutos de las Vfp fue mayor con la fertilización de

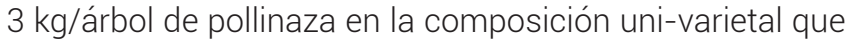
en la bi-varietal (Cuadro 1); sin embargo, sin pollinaza la firmeza fue mayor en la composición bi-varietal que en la univarietal (Figura 4B). Este incremento en la firmeza de los frutos con la aplicación de estiércol ha sido reportado por otros autores (Milošević et al., 2013).

La pollinaza disminuyó la acidez del fruto de 1.5 a $1.1 \%$ de
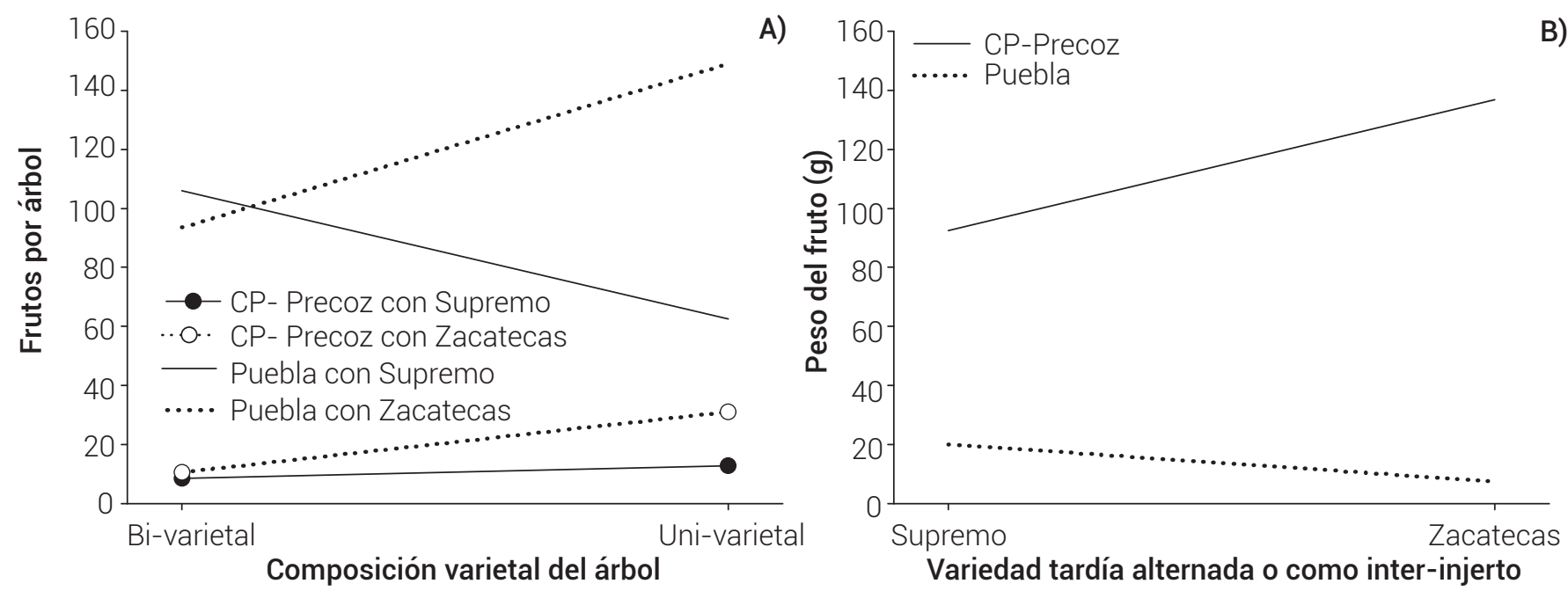

Figura 3. Efecto en variedades de floración precoz de duraznero, A) de la interacción composición varietal del árbol con la variedad tardía sobre el número de frutos por árbol y B) de la variedad tardía sobre el peso del fruto. 
la Vfp Puebla en la composición bi-varietal y la incrementó de 1.0 a 1.2 en la composición uni-varietal (Figura 5). El fenotipo de los portainjertos puede definir parámetros de calidad de frutos de las variedades injertadas, tales como la acidez (Iglesias et al., 2019).

El índice de color de los frutos en las Vfp, CP-Precoz y Puebla, fue menor en los árboles bi-varietales que en los uni-varietales con la fórmula 60-15-60 g de N- $\mathrm{P}_{2} \mathrm{O}_{5}-\mathrm{K}_{2} \mathrm{O} /$ árbol/año, pero se incrementó con la fórmula 90-30-90 g de $\mathrm{N}-\mathrm{P}_{2} \mathrm{O}_{5}-\mathrm{K}_{2} \mathrm{O} /$ árbol/año (Figura 6A y 6B). El índice de color del pericarpio de los frutos en las variedades injertadas se incrementa con portainjertos (Marcon Filho et al., 2019; Webster y Palmer, 2017) y con fertilización NPK (Minas et al., 2018; Rubio Ames et al., 2020).

Con respecto a ${ }^{\circ}$ Brix, los frutos de la Vft Supremo tuvieron menores valores en los árboles bi-varietales que en los uni-varietales; sin embargo, éstos se incrementaron de 10.6 a 11.94 al adicionar $3 \mathrm{~kg}$ de pollinaza/árbol/año (Figura 7A) y de 11.1 a 12.6, ya sea injertado o alternado con Puebla, al aumentar la dosis de fertilización de 60-

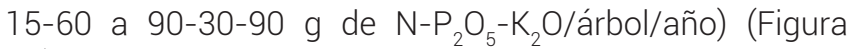
7B). Los portainjertos incrementan la concentración de los sólidos solubles totales (Font i Forcada et al., 2019) con fertilización de pollinaza y N- $\mathrm{P}_{2} \mathrm{O}_{5}-\mathrm{K}_{2} \mathrm{O}$ (Narayan et al., 2016) y mejoran otros parámetros de calidad, sobre todo con la fertilización potásica y nitrogenada (Kandil et al., 2010; Minas et al., 2018).

\section{CONCLUSIONES}

La composición bi-varietal Puebla injertada sobre Supremo tuvo el mayor rendimiento y eficiencia de rendimiento; en esta composición, Puebla tuvo el mayor peso y tamaño de frutos. La composición bi-varietal y la fertilización con la fórmula 90-30-90 g N-P $\mathrm{O}_{5}-\mathrm{K}_{2} \mathrm{O} / a$ rbol/ año aumentaron la concentración de sólidos solubles

Cuadro 1. Ecuaciones de regresión de variables del rendimiento y calidad del fruto de las variedades con floración precoz y tardía de duraznero que describen el efecto de los factores de estudio.

\begin{tabular}{|c|c|c|c|}
\hline Floración & Variable & Ecuación & $\mathrm{R}^{2}$ \\
\hline & Diámetro ecuatorial $(\mathrm{cm})=$ & $5.97744-0.35181 \vee f p-0.12131 \vee f t \vee f p$ & 0.751 \\
\hline \multirow[t]{3}{*}{ Precoz } & Acidez titulable (\% de ácido málico) = & $0.95087-0.05850$ Cva +0.26288 Vfp +0.06837 Cva P & 0.780 \\
\hline & Índice de color $\mathrm{O}^{\circ} \mathrm{Hue}=$ & $70.16789+8.21024 \mathrm{Vfp}-2.4946$ Cva F & 0.686 \\
\hline & Rendimiento $(\mathrm{kg} /$ árbol) $=$ & $8.43937-6.58875 \mathrm{Vft}-1.58563 \mathrm{Vft} \mathrm{Vfp}-2.09062 \mathrm{Vft} F-1.91563 \mathrm{Vfp} F$ & 0.918 \\
\hline \multirow[t]{2}{*}{ Tardía } & $\mathrm{ER}\left(\mathrm{kg} \mathrm{cm}^{-2}\right.$ del área del tronco $)=$ & $0.21156-0.19656 \mathrm{Vft}-0.04667 \mathrm{Vft} F$ & 0.716 \\
\hline & Firmeza $\left(\mathrm{kg} \mathrm{cm}^{-2}\right)=$ & $0.46531+0.19969 \mathrm{Vft}$ & 0.865 \\
\hline
\end{tabular}

ER: eficiencia del rendimiento, Cva (composición varietal del árbol): bi-varietal o uni-varietal, $\vee f t$ (variedad con floración tardía): Supremo o Zacatecas, Vfp (variedad con floración precoz): CP-Precoz o Puebla, F (fertilizante de NPK): 60-15-60 ó 90-30-90 g de N- $\mathrm{P}_{2} \mathrm{O}_{5}-\mathrm{K}_{2} \mathrm{O} / \mathrm{á}_{\mathrm{rbol}}$ /año) y P (pollinaza): 0 ó 3 kg/árbol/año.
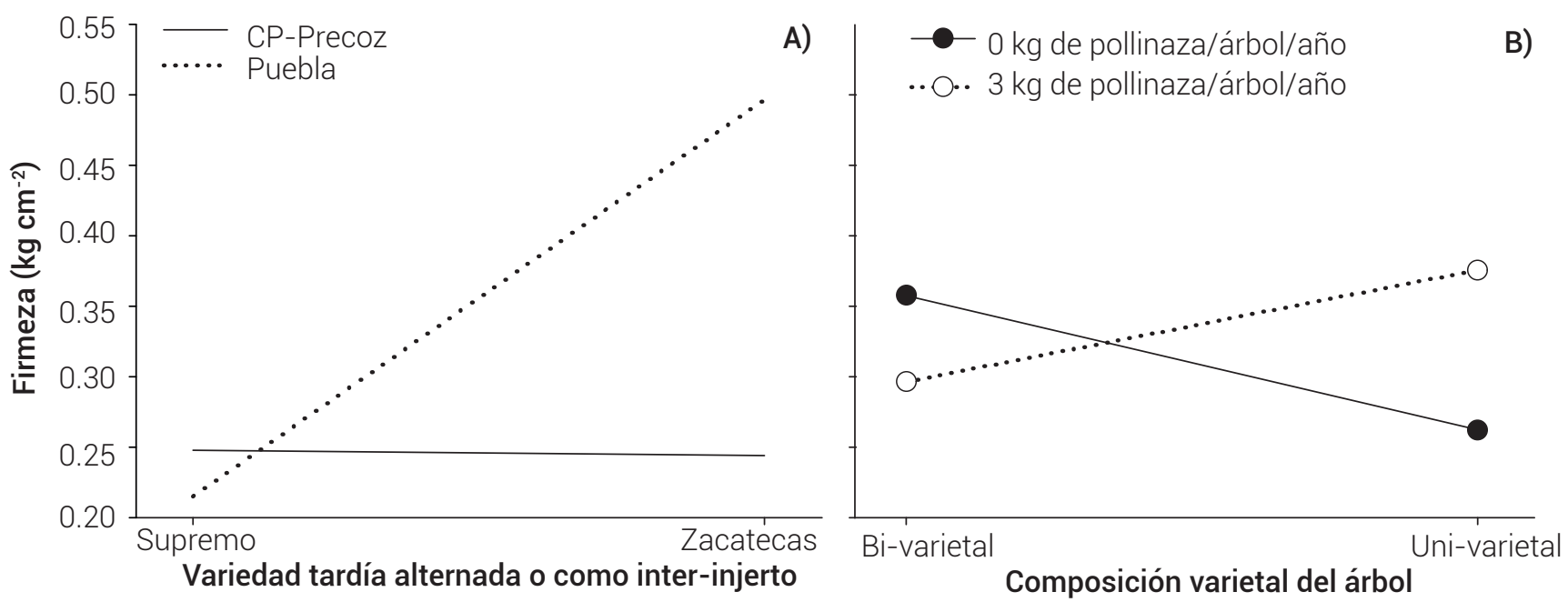

Figura 4. Efecto de: A) la variedad de floración tardía y B) de la interacción composición varietal del árbol con pollinaza sobre la firmeza del fruto de durazneros de floración precoz. 


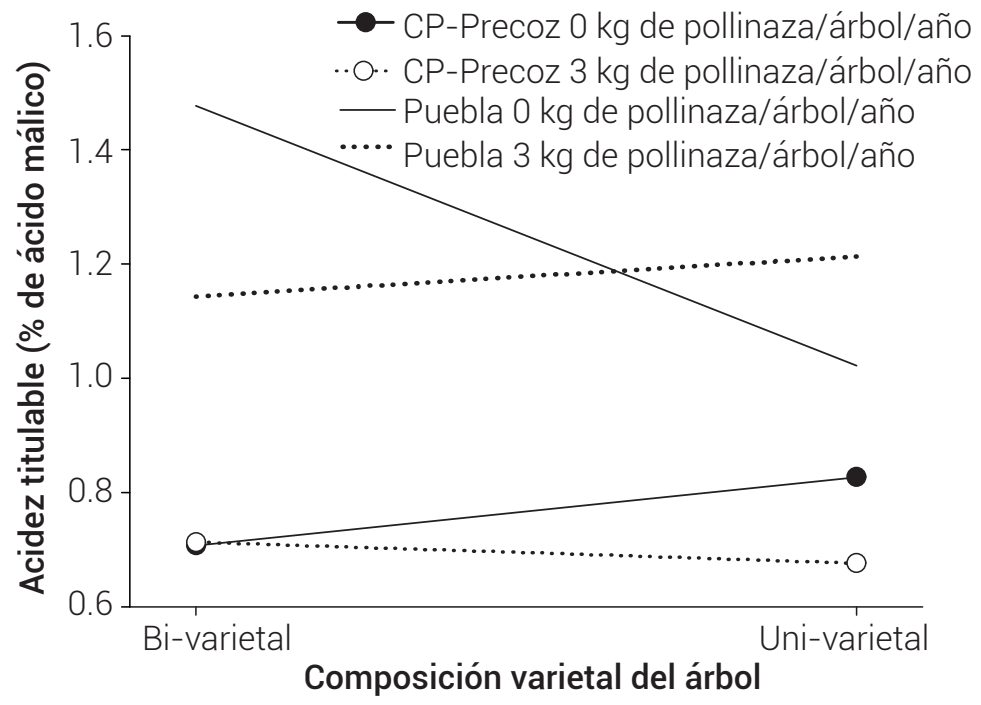

Figura 5. Efecto de la interacción composición varietal del árbol con la pollinaza sobre la acidez titulable del fruto de los durazneros con floración precoz.
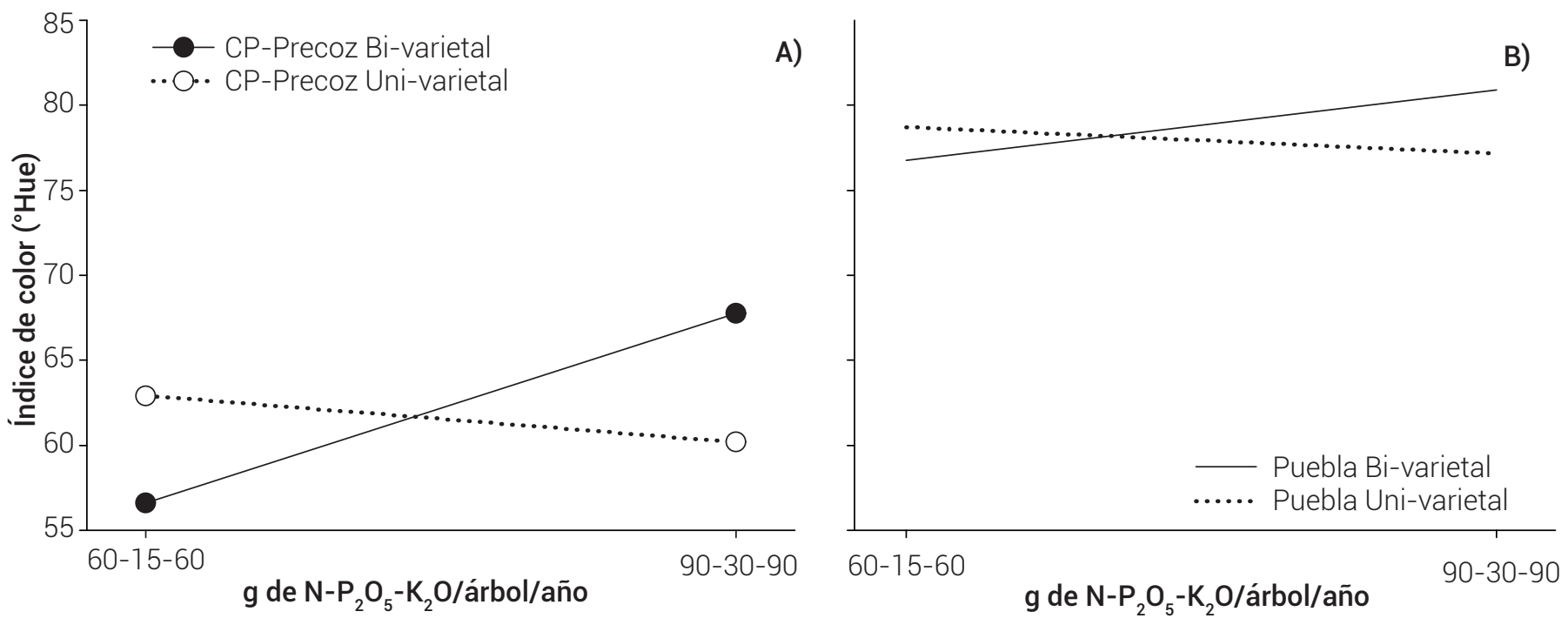

Figura 6. Efecto en el índice de color del fruto, de durazneros con floración precoz, de la interacción fertilizante $\mathrm{N}-\mathrm{P}_{2} \mathrm{O}_{5}-\mathrm{K}_{2} \mathrm{O}$ con composición varietal del árbol: A) en CP-Precoz y B) en Puebla.

totales en los frutos de Supremo y el índice de color en los de Puebla; además, la interacción composición bivarietal del árbol con el abonado de pollinaza (3 kg/árbol/ año) incrementó los sólidos solubles totales en los frutos de Supremo y disminuyó la firmeza y la acidez en los de Puebla. La alternativa de usar durazneros compuestos (bivarietales) de una variedad de floración tardía y una precoz, en fertilización adecuada con $\mathrm{N}-\mathrm{P}_{2} \mathrm{O}_{5}-\mathrm{K}_{2} \mathrm{O}$ y pollinaza tiene potencial para incrementar el rendimiento y la calidad de los frutos en el sistema MIAF en un ambiente con incidencia de heladas de radiación.

\section{BIBLIOGRAFÍA}

AOAC, Association of Official Agricultural Chemists (1990) Methods of Analysis. 15th edition. Association of Official Agricultural Chemists. Washington, D.C., USA. 1023 p.

Govaerts B., M. G. Barrera-Franco, A. Limón-Ortega, P. Muñoz-Jiménez, D. K. Sayre y J. Deckers (2008) Clasificación y evaluación edafológica de tres sitios experimentales del altiplano central de México. Tropicultura 26:2-9.

Font i Forcada C. F., G. Reig, R. Giménez, P. Mignard, L. Mestre and M. Á. Moreno (2019) Sugars and organic acids profile and antioxidant compounds of nectarine fruits influenced by different rootstocks. Scientia Horticulturae 248:145-153, https://doi. org/10.1016/j.scienta.2018.12.010

Iglesias I., J. Giné-Bordonaba, X. Garanto and G. Reig (2019) Rootstock affects quality and phytochemical composition of 'Big Top' nectarine fruits grown under hot climatic conditions. 

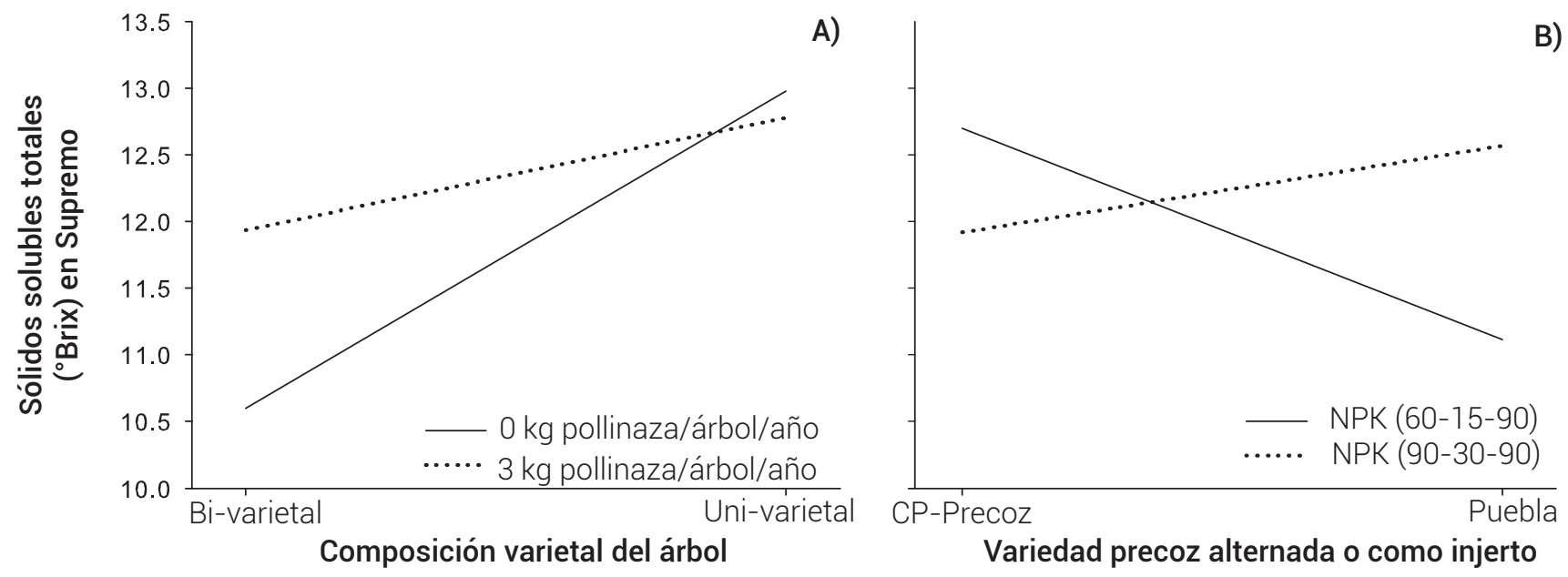

Figura 7. Efecto de las interacciones en la concentración de sólidos solubles totales del fruto de la variedad Supremo de duraznero: A) composición varietal del árbol con pollinaza y B) variedad precoz con fertilización de $\mathrm{N}-\mathrm{P}_{2} \mathrm{O}_{5}-\mathrm{K}_{2} \mathrm{O}$.

Scientia Horticulturae 256:108586, https://doi.org/10.1016/j. scienta.2019.108586

Kader A. A. and F. G. Mitchell (1989) Maturity and quality. In: Peaches, Plum and Nectarines. Growing and Handling for Fresh Market. J. H. LaRue and R. S. Johnson (eds.). University of CaliforniaDavis. Davis, CA, USA. pp:191-196.

Kandil E. A., M. I. F. Fawzi and M. F. M. Shahin (2010) The effect of some slow release nitrogen fertilizers on growth, nutrient status and fruiting of "Mit Ghamr" peach trees. Journal of American Science 6:195-201.

Kempthome 0. (1979) The Design and Analysis of Experiments. John Wiley \& Sons. New York, USA. 631 p.

Lhomme J. P., J. J. Vacher and A. Rocheteau (2007) Estimating downward long-wave radiation on the Andean Altiplano. Agricultural and Forest Meteorology 145:139-148, https://doi.org/10.1016/j. agrformet.2007.04.007

Little A. C. (1975) Off on a tangent. Journal of Food Science 40:410-411, https://doi.org/10.1111/j.1365-2621.1975.tb02213.x

Marcon Filho J. L., A. A. Kretzschmar, J. S. Hipólito, A. R. Rufato, L. Rufato and D. A. Wurz (2019) Increasing the length of EM-9 interstock enhances production efficiency in Imperial Gala apples. Revista Ceres 66:178-183, https://doi.org/10.1590/0034737X201966030003

Marra F. P., R. L. Bianco, M. L. Mantia and T. Caruso (2013) Growth, yield and fruit quality of 'Tropic Snow' peach on size-controlling rootstocks under dry Mediterranean climates. Scientia Horticulturae 160: 274-282, https://doi.org/10.1016/j.scienta.2013.06.004

Mestre L., G. Reig, J. A. Betrán and M. A. Moreno (2017) Influence of plum rootstocks on agronomic performance, leaf mineral nutrition and fruit quality of 'Catherina' peach cultivar in heavy-calcareous soil conditions. Spanish Journal of Agricultural Research 15:e0901, https://doi.org/10.5424/sjar/2017151-9950
Milošević T., N. Milošević and I. Glišić (2013) Tree growth, yield, fruit quality attributes and leaf nutrient content of 'Roxana'apricot as influenced by natural zeolite, organic and inorganic fertilisers Scientia Horticulturae 156:131-139, https://doi.org/10.1016/j. scienta.2013.04.002

Minas I. S., G. Tanou and A. Molassiotis (2018) Environmental and orchard bases of peach fruit quality. Scientia Horticulturae 235:307-322, https://doi.org/10.1016/j.scienta.2018.01.028

Narayan A., P. N. Singh, M. Brijwal and S. K. Singh (2016) Response of organic manures on quality of peach (Prunus persica Batsch) cv Florda Prince. Environment and Ecology 34:985-987.

Rubio Ames Z., J. K. Brecht and M. A. Olmstead (2020) Nitrogen fertilization rates in a subtropical peach orchard: effects on tree vigor and fruit quality. Journal of the Science of Food and Agriculture 100:527-539, https://doi.org/10.1002/jsfa.10031

Santiago-Mejía H., J. I. Cortés-Flores, A. Turrent-Fernández, E. García-Moya, M. Livera-Muñoz, H. A. Zavaleta-Mancera y V. Volke-Haller (2015) Respuesta de árboles de duraznero [Prunus persica (L.) Batsch] compuesto de dos cultivares en un ambiente con incidencia de heladas: floración y amarre de fruto. Agrociencia 49:573-592.

Webster A. D. and J. W. Palmer (2017) Pome and stone fruit. In Horticulture Production and Quality. Encyclopedia of Applied Plant Sciences. Second edition. Vol. 3. B. Thomas, B. G. Murray and D. J. Murphy (eds.). Academic Press, New York, USA. pp:193-202, https://doi.org/10.1016/B978-0-12-394807-6.00009-5

Westwood M. N. (1993) Temperate-Zone Pomology, Physiology and Culture. Timber Press, Portland, OR. 523 p.

Yano T., H. Inoue, Y. Shimizu and S. Shinkai (2002) Dry matter partitioning and carbohydrate status of 'Kawanakajima Hakuto' peach trees grafted onto different rooststocks or with an interstock at prebloom period. Journal of the Japanese Society for Horticultural Science 71:164-170, https://doi.org/10.2503/jjshs.71.164 
